\title{
Development and validation of a sensitive monoclonal antibody-based ic-ELISA for the aflatoxin $M_{1}$ in milk (Abstract)
}

\author{
Dapeng Peng \\ College of veterinary medicine, Huazhong Agricultural University, Wuhan, China
}

Corresponding author: pengdapeng@mail.hzau.edu.cn

\begin{abstract}
Aflatoxin $\mathrm{M}_{1}\left(\mathrm{AFM}_{1}\right)$, a hydroxylated metabolite of aflatoxin $\mathrm{B}_{1}\left(\mathrm{AFB}_{1}\right)$, possesses the carcinogenic, mutagenic, and toxic activities. To protect the health of animals and humans, it is clear that the need for an effective residue-monitoring programme to detect the $\mathrm{AFM}_{1}$ residues. In this study, a modified procedure was used to prepare the hapten $\mathrm{AFB}_{1}$ and $\mathrm{AFM}_{1}$ derivatives. Then, the prepared antigen $\mathrm{AFM}_{1}-\mathrm{CMO}-\mathrm{KLH}$ was used to inoculate female Balb/c mice to prepare a sensitive monoclonal antibody (mAb) against $\mathrm{AFM}_{1}$. After cell fusion and culture several times, the hybridoma cell line, 3D8, which was of the IgG1 isotype, was selected to obtain a highly sensitive mAb. The obtained 3D8 mAb displayed an $\mathrm{IC}_{50}$ value of $64.75 \mathrm{ng} \mathrm{L}^{-1}$ for $\mathrm{AFM}_{1}$ and did not exhibit measurable cross-reactivity with other aflatoxins and antibiotics. Based on this mAb, an indirect competitive enzyme-linked immunosorbent assay (ic-ELISA) was established that utilizes simple sample preparation and clean-up methods. The decision limit (CC $\alpha, \alpha=1 \%)$, detection capability $(\mathrm{CC} \beta, \beta=5 \%)$, and LOQ value for the $\mathrm{AFM}_{1}$ matrix calibration method were $24 \mathrm{ng} \mathrm{L}^{-1}, 27.5 \mathrm{ng} \mathrm{L}^{-1}$, and $35 \mathrm{ng} \mathrm{L}^{-1}$ in the milk matrices, respectively. The $\mathrm{AFM}_{1}$ recovery ranged from $85.3 \%$ to $107.6 \%$. The CVs were less than $13.8 \%$. A positive correlation ( $\mathrm{r}>0.99$ ) was observed between the ic-ELISA and HPLC-MS/MS results. This ic-ELISA would be a useful tool for screening the $\mathrm{AFM}_{1}$ residues in milk.
\end{abstract}

Keywords: Monoclonal antibody (mAb), Aflatoxin $\mathrm{M}_{1}$, milk. 
